\section{Improving the patient journey for Aboriginal and Torres Strait Islander people with acute coronary syndromes}

To THE EDITOR: It has been estimated that "levelling the playing field" in terms of cardiovascular health alone could narrow the lifeexpectancy gap between Indigenous and non-Indigenous Australians by 6.5 years. $^{1}$

While there has been a particular (and important) focus on risk factor modification, and prevention and management of chronic disease, it is worth noting that, compared with other Australians, Aboriginal and Torres Strait Islander people have: - three times the rate of major coronary events

- higher death rates from coronary heart disease both in and out of hospital

- lower rates of coronary angiography, angioplasty and bypass surgery $^{2}$

- a greater likelihood of adverse outcomes in the 2 years after discharge. $^{3}$

These statistics suggest that Aboriginal and Torres Strait Islander people experiencing symptoms of acute coronary syndromes (ACS) are falling through gaps at every stage of the journey through the health care system. Access to services and the sequence of care for these people could be vastly improved.

In 2010, the Heart Foundation and Australian Healthcare and Hospitals Association (AHHA) published a report examining these disparities and providing specific recommendations to achieve better care for Aboriginal and Torres Strait Islander people throughout the patient journey. ${ }^{4}$ It acknowledged that, although quality care is being provided in some places, more research and comprehensive data collection are needed.

The Heart Foundation and AHHA are now seeking support for a national "lighthouse hospital" initiative to showcase exemplary efforts in this area and allow sharing and collaboration between participating hospitals (see http:// www.heartfoundation.org.au/ information-for-professionals/ aboriginal-health for more details).

Initiatives that successfully improve the quality of care of Aboriginal and Torres Strait Islander people with ACS generally do so by attending to one or more of four key areas:

- Cultural competence refers to both an organisation's and an individual's capacity to respect and accommodate another's set of beliefs, providing a safe and secure environment in which to deliver care.

- A focus on clinical quality improvement allows for each step in the care pathway to be monitored and adapted.

- The workforce requires training in dealing with other cultures and quality improvement, and it needs to be of adequate number (including Aboriginal health staff).

- Overarching governance and accountability, including genuine engagement of management, is crucial.

If your hospital has implemented a program to improve the care of Aboriginal and Torres Strait Islander people with ACS, please help us collate the evidence and advocate for an Australian lighthouse hospital initiative by emailing the details to kelley.odonohue@heartfoundation. org.au.

Robert D Grenfell Director, Clinical Issues' Vicki Wade National Leader, National Aboriginal Health Unit ${ }^{2}$

Eleanor Clune Senior Project Officer' Kelley O'Donohue Senior Project Officer ${ }^{3}$ Prue Power Chief Executive Officer ${ }^{4}$ 1 Heart Foundation, Melbourne, VIC. 2 Heart Foundation, Sydney, NSW.

3 Heart Foundation, Brisbane, QLD.

4 Australian Healthcare and Hospitals Association, Canberra, ACT.

kelley.odonohue@heartfoundation.org.au

Competing interests: $N$ No relevant disclosures. doi: 10.5694/mjal2.10443

1 Australian Bureau of Statistics. Mortality and morbidity: mortality of Aboriginal and Torres Strait Islander peoples. In: Australian social trends, 2002. Canberra: ABS, 2002. (ABS Cat. No. 4102.0.)

2 Mathur S, Moon L, Leigh S. Aboriginal and Torres Strait Islander people with coronary heart disease: further perspectives on health status and treatment Canberra: Australian Institute of Health and Welfare, 2006. (AlHW Cat. No. CVD 33; Cardiovascular Disease Series No. 25.)

3 Brown A. Acute coronary syndromes in Indigenous Australians: opportunities for improving outcomes across the continuum of care. Heart Lung Circ 2010; 19: 325-336.

4 National Heart Foundation of Australia and Australian Healthcare and Hospitals Association. Better hospital care for Aboriginal and Torres Strait Islander people experiencing heart attack. 2010. http://www.heartfoundation.org.au/ SiteCollectionDocuments/A-ATSI-BetterHospital-Care-20110812.pdf (accessed Jun 2012).
Australians, Aboriginal and

Torres Strait

Islander people

have three

times the rate of

major coronary

events

Grenfell et al 